\title{
Clinical and Laboratory Follow-up of Children with COVID-19
}

\author{
Marita Antoniadi $^{1}$ (D) Dimitra-Irinna Vitoratou ${ }^{1} \cdot$ Kyriaki Chorianopoulou $^{2} \cdot$ Konstantina Giannakopoulou $^{2}$. \\ Efstathia Staikou ${ }^{2} \cdot$ Patra Koletsi $^{1} \cdot$ Stavroula Kostaridou ${ }^{1}$
}

Received: 17 October 2021 / Accepted: 14 January 2022 / Published online: 15 February 2022

(c) Dr. K C Chaudhuri Foundation 2022

To the Editor: During the current dynamic SARS-CoV-2 pandemic, emerging confounding evidence suggest that despite overall favorable outcome, persisting symptoms, defined as postacute or long COVID-19, have been observed in the pediatric population [1-4].

We aimed to prospectively follow up patients $<16$ y old, diagnosed with COVID-19 by RT-PCR, three months post diagnosis to explore for any sequelae associated with COVID-19.

One hundred sixty-eight patients were identified from May 2020 to April 2021; of which, 106 were finally included (median age $8 \mathrm{y}$ ); $44.3 \%$ were male and $10.4 \%$ were obese. Six had underlying chronic conditions (chronic lung disease $=4$, metabolic disorder $=1$, autoimmune disease $=1$ ); $15.1 \%$ were hospitalized (mean age $5.8 \mathrm{y}$ ); of which, 1 was admitted to high-dependency unit for monitoring. No patients suffered critical disease and none died. Mean follow-up time was 83 d. However, 3 patients presented earlier (at 52, 56, and $58 \mathrm{~d}$, respectively), diagnosed with MIS-C. Underweight and younger children were more frequently hospitalized $(p=0.07$ and $p=0.044)$. Sex was not associated with COVID-19 severity $(p=0.423)$.

At follow-up, ECG was performed in all patients with no new-onset electrocardiographic disturbances. Of the laboratory workup conducted, the abnormal values most frequently seen were prolonged APTT $(82,7 \%)$, followed by high monocyte absolute count $(74,5 \%)$. Troponin was normal in all subjects except MIS-C cases. A negative association between age group and IgG COVID-19 antibody levels was observed $(p=0.03)$, whereas no association was

Marita Antoniadi and Dimitra-Irinna Vitoratou contributed equally to this manuscript

Marita Antoniadi

marita_ant@yahoo.gr

1 Department of Pediatrics, Penteli Children's Hospital, Athens 15236, Greece

2 Department of Biopathology, Penteli Children's Hospital, Athens, Greece found between disease severity or weight status and IgG levels ( $p=0.36$ and $p=0.38$ ). When questioned for persistent symptoms, 4 patients 8 to $15 \mathrm{y}$ of age, reported fatigue and a female adolescent complained of anxiety since admission.

In our cohort, three months post-COVID-19 infection, children were mostly asymptomatic with no organ-specific damage, and showed mild disease sequelae mostly described as chronic fatigue. We aim to contribute this experience in the quest for the optimal timing for pediatric patients' reevaluation after COVID-19 infection.

\section{Declarations}

Conflict of Interest None.

\section{References}

1. Dong Y, Mo X, Hu Y, Qi X, Jiang F, Jiang Z, Tong S. Epidemiology of COVID-19 among children in China. Pediatrics. 2020;145:e20200702.

2. Badal S, Thapa Bajgain K, Badal S, Thapa R, Bajgain BB, Santana MJ. Prevalence, clinical characteristics, and outcomes of pediatric COVID-19: A systematic review and meta-analysis. J Clin Virol. 2021;135:104715.

3. Osmanov IM, Spiridonova E, Bobkova P, et al. Risk factors for long covid in previously hospitalised children using the ISARIC global follow-up protocol: a prospective cohort study. Eur Respir J. 2021. https://doi.org/10.1183/13993003.01341-2021.

4. Michelen M, Manoharan L, Elkheir N, et al. Characterising long COVID: a living systematic review. BMJ Glob Health. 2021;6:e005427

Publisher's Note Springer Nature remains neutral with regard to jurisdictional claims in published maps and institutional affiliations. 